\title{
Bikram Yoga as a Countermeasure of Bone Loss in Women
}

\author{
Apurba Mukherjee ${ }^{1}$, Prithwis Mukherjee ${ }^{1}$, Robert R. Rude ${ }^{2}$ \\ ${ }^{1}$ Bikram's Yoga College of India, Los Angeles, USA \\ ${ }^{2}$ Keck School of Medicine, University of Southern California and Orthopedic Hospital of Los, Los Angeles, USA \\ E-mail:apurba@IEEE.org
}

Received May 28, 2010; revised June 10, 2010; accepted June 20, 2010

\begin{abstract}
The purpose of this pilot study was to observe whether Bikram Yoga training helps bone growth or arrest bone loss in women. In this study, the bone mineral density (BMD) was measured at the hip, spine and whole body for a group of 14 pre-menopausal women (11 Caucasians, 2 Asians and 1 African American). These 14 women had participated in Bikram Yoga classes (26 yoga postures) at least 3 times a week for 3 or more years. DEXA scans were used to measure BMD at the lumbar spine, hip and the whole body. In addition, the Z-scores were calculated for each subject at these three locations. The study results indicate that the BMD at these body areas of this group of women is generally above the mean BMD expected for normal, healthy, women of comparable age and ethnicity. Overall, the study findings suggest that the intervention of Bikram Yoga training may be beneficial for skeletal health and could prevent bone loss.
\end{abstract}

Keywords: Osteoporosis, Bone Mineral Density (BMD)

\section{Introduction}

A pilot study was initiated to examine whether prevention/recovery from bone loss is possible through Bikram Yoga postural exercise intervention. Bone loss is a serious medical problem [1], and an estimated 200 million adults (both men/women) have this condition worldwide. The sharp decline of production of the hormone estrogen following menopause contributes greatly to bone loss for the elderly women population. Bone loss also results from joint disuse and reduced movements. The study was aimed to explore the possibility of using Bikram Yoga postural exercise intervention as a possible means of prevention/recovery for bone loss.

In general, the skeleton reaches its peak bone density for people between the ages of 20 and 30, and after age 35 , the body begins to lose more bone than the body can rebuild. According to the Surgeon General's Report (2004) [2], there are over 10 million Americans over the age of 50 with osteoporosis and an additional 34 million with low bone mass or osteopenia. An estimated 1.5 million Americans suffer from fractures related to bone disease every year. In addition, about two-thirds of the people suffering from osteoporosis are women, as they abruptly lose the bone-protecting estrogen hormone at menopause [3]. However, men also suffer from osteoporosis as they age. In fact, one in four elderly men in
America suffers from osteoporotic fracture in older age.

Bones are living tissues and are continually broken down and rebuilt in human bodies. Consisting of $75 \%$ minerals, (like calcium and phosphorous), and 25\% gelatin matrix of water and collagen, bones continue to be reshaped and renewed all the time. While bones appear solid they can be quite flexible and strong at the same time.

There is a delicate balancing act that goes on daily with minerals and human bones. A healthy body reabsorbs bone at the same rate as new bone growth occurs. Specialized cells called osteoclasts will remove bone while osteoblasts build bone. This cycle continues over and over.

Bikram Yoga, which is a certified form of exercise by the State of California [4], is suitable for the young as well as the elderly people. Although Bikram Yoga is widely practiced in over 1500 studios in USA, Canada, Europe, Japan, Australia, Thailand, India and many other countries around the world, there have been limited efforts to scientifically study and document the far reaching benefits of Bikram Yoga to the mind and body.

\section{Study Methods}

All study subjects were enrolled by Bikram's Yoga College of India in Los Angeles and they signed informedconsent forms for Bone Mineral Density (BMD) meas- 
urements at the Orthopedic Hospital in Los Angeles. The subjects were Bikram Yoga teachers and they consistently practiced and taught Bikram Yoga on a daily basis. The Bikram Yoga exercise intervention is a 90-minute protocol of specially designed sequence of 26 postures and two breathing exercises, which can be practiced by men or women of any age. These are static isometric postural exercises and there are no dynamic movements. These exercises, which involve both the mind and the body, collectively stimulate all the vital organs of the body. The details of the different 26 postures are described in reference [5].

Bikram Yoga is a standardized yoga program that is designed to scientifically warm up and stretch muscles, ligaments and tendons. In all parts of the world, Bikram Yoga teachers train participants to perform the same 26 postures, in the same sequence, at 105 degrees Fahrenheit. Bikram Yoga is a standardized system of yoga and no deviation is allowed between classes. In fact, all Bikram Yoga teachers are trained and certified by the Bikram's Yoga College of India. All certified teachers also undergo periodic evaluations and their teaching certificates are revalidated every three years. The 26 exercises systematically move fresh, oxygenated blood to each organ [6].

Bone mineral density (BMD) was determined utilizing a Hologic bone densitometer. Bone mass is commonly expressed as Z-scores as defined below.

Z-score: The Z-scores are calculated by comparing patient's BMD with the corresponding BMD of women of the same age. sex and race. Z-scores are calculated as follows:

Z-score $=($ BMD-Expected BMD $) / S$. D.

$\mathrm{BMD}=$ patient's BMD

Expected BMD = BMD of someone of the same age, sex and race.

S. D = Standard Deviation corresponding to the above “expected BMD”.

Z-score is a statistical concept.

The Z-score compares the BMD to age, gender and ethnicity of the individual. Data presented here therefore are the Z-scores.

The Z-score value lower than -2.0 is a warning sign that the patient has less bone mass (and/or may be losing more rapidly) than expected for someone of corresponding age, sex and race.

\section{Results}

The bone mass density (BMD) for each subject was calculated for the lumbar spine, hip, and whole body. Table 1 shows the BMD data, in addition to basic demographic data, for each of the 14 study participants.

Figure 1 demonstrates the standard interpretation of Z-score for the whole body and Figure 2 demonstrates the Z-scores for the study population.

Figure 2 demonstrates that whole body calcium of all the 14 subjects are in the above average calcium group of population. In addition, Figures 1 and 2 show that 6 out of 14 subjects have bone density higher than $84 \%$ (or in the top $16 \%$ ) of the population.

Table 1. Age, yoga experience and DEXA scan BMD data.

\begin{tabular}{|c|c|c|c|c|c|c|}
\hline & Age (years) & Total \# of yoga classes & Years of Yoga practice & Z-score (spine) & Z-score (hip) & Z-score (whole body) \\
\hline Subject 1 & 46 & 450 & 3 & 2.0 & 1.7 & 2.1 \\
\hline Subject 2 & 47 & 800 & 4 & 1.2 & 0.6 & 1.2 \\
\hline Subject 3 & 49 & 600 & 4 & 0.6 & -0.5 & 0.2 \\
\hline Subject 4 & 39 & 975 & 4 & -0.3 & 0 & -0.3 \\
\hline Subject 5 & 41 & 700 & 4 & -0.8 & -0.5 & -0.7 \\
\hline Subject 6 & 47 & 675 & 3 & -0.4 & 0.4 & 0.7 \\
\hline Subject 7 & 31 & 975 & 4 & 2.1 & 1.5 & 2.6 \\
\hline Subject 8 & 50 & 950 & 4 & 1.4 & 0.9 & 3.7 \\
\hline Subject 9 & 47 & 950 & 4 & -1.3 & -0.9 & -1.1 \\
\hline Subject 10 & 45 & 900 & 4 & -0.5 & -0.4 & 0.3 \\
\hline Subject 11 & 41 & 525 & 3 & -0.8 & -0.1 & -0.3 \\
\hline Subject 12 & 49 & 525 & 3 & 1.0 & 0.5 & 1.9 \\
\hline Subject 13 & 45 & 700 & 4 & -0.5 & -0.2 & 0 \\
\hline Subject 14 & 44 & 600 & 3 & -0.6 & 0.6 & 1.6 \\
\hline Mean & 44 & 738 & 3.6 & 0.22 & 0.26 & 0.85 \\
\hline
\end{tabular}




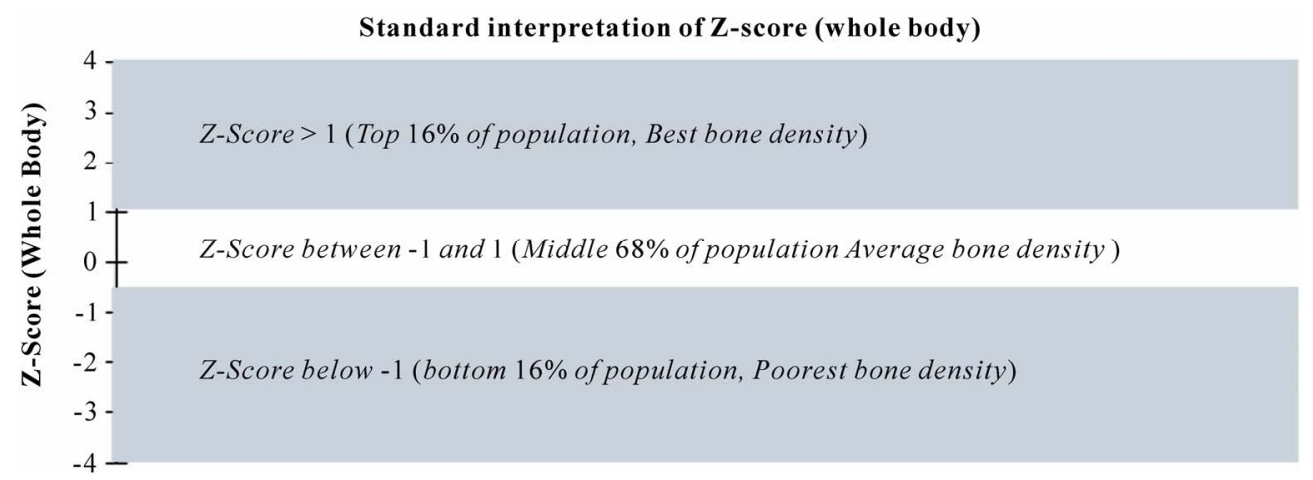

Figure 1. Standard interpretation of Z-score (whole body).

Z-score (whole body) by Number of Yoga Classes

In addition, 6 out of the 14 yoga teachers have bone density better than $84 \%$ of the population

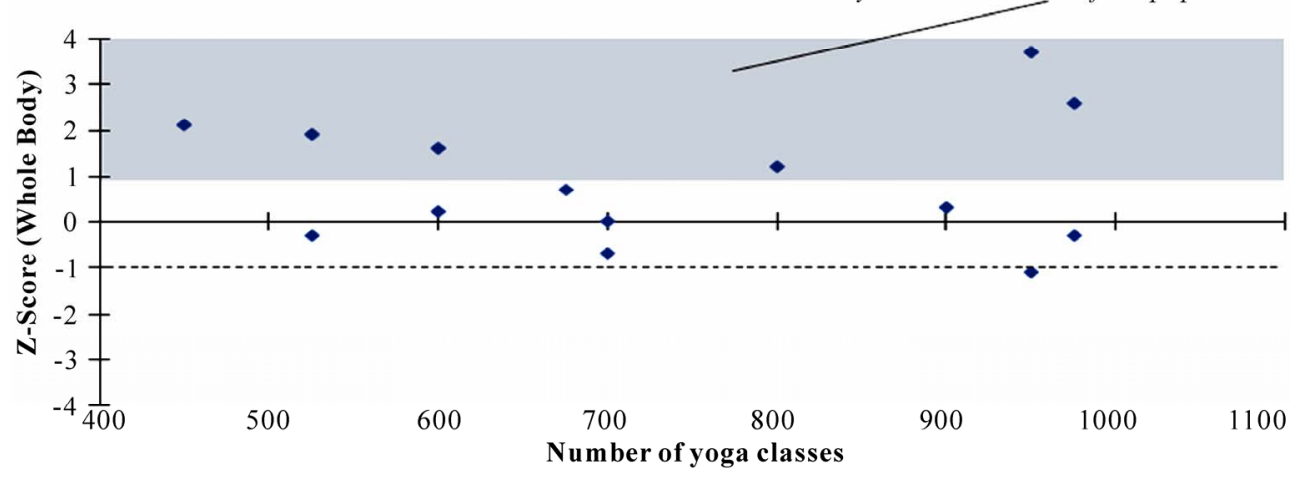

Figure 2. Z-score (whole body) by the number of Bikram Yoga classes.

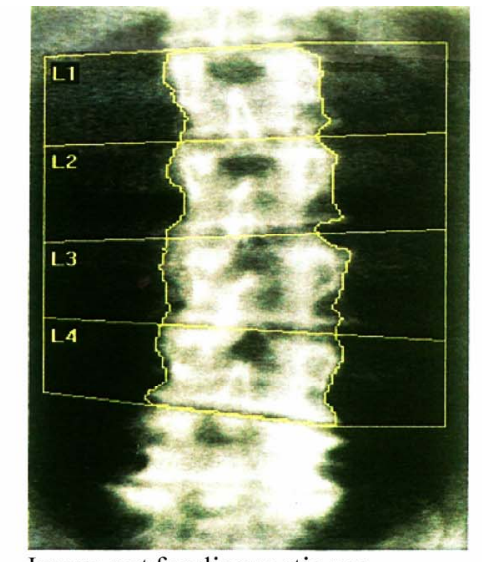

$\begin{array}{ll} & \text { Lumbar Spine } \\ \text { Operator: } & \text { MH } \\ \text { Model: } & \text { Delphi A (S/N 70345) } \\ \text { Comment: } & \end{array}$

Image not for diagnostic use $\mathrm{k}=1.136, \mathrm{~d} 0=44.8$ $116 \times 139$

Comment:

\author{
Lumbar Spine \\ Delphi A (S/N 70345)
}

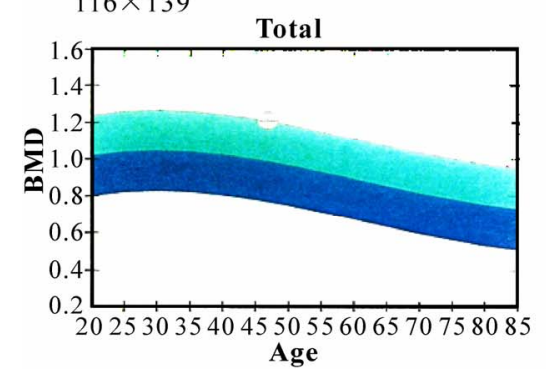

DXA Results Summary:

\begin{tabular}{|lccccccc|}
\hline Region & $\begin{array}{c}\text { Area } \\
\left(\mathbf{c m}^{2}\right)\end{array}$ & $\begin{array}{c}\text { BMC } \\
(\mathbf{g})\end{array}$ & $\begin{array}{c}\text { BMD } \\
\left(\mathbf{g} / \mathbf{c m}^{2}\right)\end{array}$ & $\begin{array}{c}\text { T- } \\
\text { Score }\end{array}$ & $\begin{array}{c}\text { PR } \\
(\mathbf{\%})\end{array}$ & $\begin{array}{c}\text { Z- } \\
\text { Score }\end{array}$ & $\begin{array}{c}\text { AM } \\
(\mathbf{\%})\end{array}$ \\
L1 & 14.06 & 15.15 & 1.077 & 1.4 & 116 & 1.9 & 123 \\
L2 & 15.97 & 17.99 & 1.126 & 0.9 & 110 & 1.4 & 116 \\
L3 & 17.16 & 20.13 & 1.173 & 0.8 & 108 & 1.4 & 115 \\
L4 & 16.10 & 23.34 & 1.449 & 3.0 & 130 & 3.6 & 138 \\
Total & $\mathbf{6 3 . 3 0}$ & $\mathbf{7 6 . 6 1}$ & $\mathbf{1 . 2 1 0}$ & $\mathbf{1 . 5}$ & $\mathbf{1 1 6}$ & $\mathbf{2 . 0}$ & $\mathbf{1 2 3}$ \\
\hline
\end{tabular}

Total BMD CV 1.0\%, ACF $=1.026, \mathrm{BCF}=1.014, \mathrm{TH}=6.721$

WHO Classification: Normal

Fracture risk: Not Increased

\title{
HOLOGIC
}

Physician's Comment:

Figure 3. Photo of a DEXA scan (lumbar spine). 
Figure 3 shows a typical DEXA scan photo from the HOLOGIC bone densitometer at the Orthopedic Hospital in Los Angeles.

\section{Discussion}

The results demonstrate that the mean bone mineral density of all 14 subjects at the lumber spine, total hip, and the whole body are above average considering their ages and ethnicities. This is most notable for the total body calcium where the Z-score (whole body) is nearly one standard deviation above the mean. The data suggests that an intervention trial of Bikram Yoga exercise activity may arrest bone loss in women.

\section{Practical Applications}

The practice of Bikram Yoga may provide another means to stabilize bone mass and prevent bone loss and osteoporotic fractures.

\section{Acknowledgements}

This study was supported by Bikram's Yoga College of India in Los Angeles. The contribution of Travis Liggett in the early stage of study in obtaining the Institutional
Review Board (IRB) approval is gratefully acknowledged. In addition the contribution of Anita Mukherjee (Stanford University) in statistical analysis and developing Figures $1 \& 2$ from the data of this article are acknowledged.

\section{References}

[1] B. W. Watts, B. Ettinger and M. S. LeBoff, "Perspective, FRAX Facts,” Journal of Bone and Mineral Research, Vol. 24, 2009, pp. 975-979.

[2] U. S. Dept, "Bone Health and Osteoporosis," Health and Human Services, Washington, D.C., 2004. http://www. surgeongeneral.gov

[3] "Risk and Benefits of Estrogen Plus Progestin in Healthy Post Menopausal Women," Journal of the American Medical Association, Vol. 288, No. 3, 17 July 2002, pp. 321-333.

[4] State of California License to Bikram Choudhury for Bikram Yoga College of India, 2005.

[5] B. Choudhury, "Bikram’s Beginning Yoga Classes," Penguin Putnam Inc., New York, 2000.

[6] B. Choudhury, "Bikram Yoga,” Harper Collins Publishers, New York, 2007, p. 84. 